\title{
Dynamic Power Control and Delay-Aware Forwarder Selection in mostly-off WSNs
}

\author{
Rahim Kacimi and Zoubir Mammeri \\ IRIT-UPS, University of Toulouse, Toulouse, France. \\ \{kacimiImammeri\}@irit.fr
}

\begin{abstract}
During the past decade, lifetime maximizing in wireless sensor networks was the major concern in this research area. Thus, "mostly-off" sensor nodes alternate between active and sleep states in many solutions nowadays. Unfortunately, these cycles involve strong sleep latencies which are the major limitation leading the solutions to suffer severe end-to-end delay degradation. Our objective in this paper is to investigate the delay issue in low duty-cycled WSN. We propose a delay-aware mechanism where each node having packets to send must choose a forwarder whose next active slot is the closest. Besides, we combine power controlled unicast transmissions with dynamic forwarder selection to better bound the communication delay and save energy. Through extensive simulations, we examine the performance of our design under different configurations and show how it outperforms the traditional routing schemes such as shortest-path algorithm.
\end{abstract}

Index Terms-Duty-cycling, transmission power control, routing, communication delay, energy consumption.

\section{INTRODUCTION}

Since it is not possible to maintain an always-awake communication backbone in low duty-cycled WSN, the end-to-end delay increases significantly. This phenomenon is due to long sleep periods of the nodes. Indeed, in low duty-cycle sensor networks, the nodes turn off their radio most of the time. Consequently, they can not always forward data whenever their previous-hop nodes need to send data.

In this paper, We study multi-hop routing with many-to-one traffic pattern in low duty-cycle WSN, where time is divided into consecutive slots grouped into equal sized frames. To send data the nodes should prefer forwarders that are upcoming to wake up in order to reduce the delay caused by the sleeping latency. On a different front, there is increasing latency when dealing with very low duty-cycles and limited number of forwarders.

We examined some proposed protocols and we noted that they are based on neighbor discovery algorithms, which are mainly operating with a single transmission power level (the maximum one). Consequently, the nodes select always the same forwarders, ie. those having the minimum distance in terms of number of hops to reach the Sink (like shortest-path routing). Such a mechanism is admittedly a helpful way to reduce the end-to-end delay in "mostly-on" WSN. However, this operating mode consumes more energy especially during neighbor-discovery (collisions, interference). Furthermore, since they select only one closest forwarder, the nodes have fewer opportunities to send their packets as soon as possible.
In this paper, we argue in favor of a new mechanism where the nodes gradually adapt their transmission power level and select any forwarder closer than them to the sink. The reminder of this paper is organized as follows: In the next section, we discuss previous related work. We propose a delay-aware routing scheme in section III. In section IV, the performance of our solution is evaluated and compared to other protocols through simulation. Section V concludes this paper.

\section{RELATED WORK}

WSN protocols use different techniques to efficiently control the energy consumption, and most of them employ the duty-cycling one. Therefore, satisfying a packet transfer in bounded delays is obviously a complicated task. However, there are still important efforts which lie in the same research topic of our contribution.

First, it should be noted that various duty-cycling approaches have been proposed at the MAC layer. SCP [6] serves as an example. Designed for ultra-low duty-cycle WSN, it uses low-power listening technique to extend the network lifetime and adapts to heavy traffic and streams data in multihop networks to reduce latency.

In [2], authors propose three different approaches to provide real-time guarantee of communication delay. They start by increasing the duty-cycle at individual node, then they propose a scheme on placement of sink nodes and finally they present a hybrid approach which balances between cost and efficiency on bounding communication delay.

More recently, another category of related works focus on timely and reliable data forwarding in sensor networks. For instance, in [1], Gu and He introduce "dynamic switch-based forwarding" concept to optimize the expected data delivery ratio and the communication delay for low-duty-cycle WSN under unreliable communication links. Later, Wang and Liu [5] consider the scenario of providing a network-wide broadcast service and propose to make effective use of local broadcast of wireless medium, achieving a balance between efficiency and latency with coverage guarantees.

Different from the aforementioned work, in our contribution we consider "many-to-one" traffic pattern in low-duty-cycle WSN. Our solution combines power controlled unicast transmissions with dynamic forwarder selection to better bound the communication delay and save energy.

Our contribution lies also in the transmission power control (TPC) topic. There have been works investigating this research 
area. Among them, ATPC [3] may probably serves as the most relevant example. With ATPC, the authors employ a feedbackbased TPC algorithm to dynamically maintain individual link quality. To this end, each node builds a model describing the correlation between transmission power and link quality of its neighbors.

\section{PROPOSED SCHEME}

In this work, we consider a multi-hop wireless sensor network, where homogeneous sensor nodes are randomly deployed. In this section, we detail our design assuming that:

- The whole network is connected at least with the maximum transmission power.

- The clocks of the nodes are synchronized in the neighborhood.

To clarify our design, we introduce the following definitions:

Node Level $\left(\mathcal{N}_{\mathcal{L}}\right)$ : this counter represents the distance in terms of number of hops between a node and the final destination (sink). It is calculated during the initialization phase. Thus, it follows from the layering of the network topology. The nodes may have more than one level. Indeed, according to the used transmission power, a node level may vary.

Duty-Cycle Schedule $(\mathcal{D C S})$ : all the nodes adopt low dutycycle schedules which are normally periodic [4]. As shown in Fig. 3, we consider a common period $T_{\max }$, it is the set of the overall time slots where the nodes may choose randomly various active slots. We assume that a time slot corresponds to the sufficient duration to send/receive a packet and receive/send an acknowledgment. Let $\mathcal{D C S} \mathcal{S}^{i}=\left\{s_{1}^{i}, s_{2}^{i} \ldots, s_{N}^{i}\right\}$ denote the active duty-cycle schedule of node $i$, where $s_{k}^{i}$ is the $k^{\text {th }}$ wake-up time of node $i$ during $T_{\max }$. For example node $i$, in Fig. 1, is scheduled to be active in the times slots 7, 46 and 99 in $T_{\max }=100$ and the duty-cycle is equal to $3 \%$, thus $\mathcal{D C S}^{i}=\{7,46,99\}$.

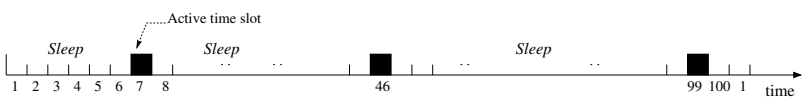

Figure 1. Periodic schedule with a duty-cycle of $3 \%$.

Set of forwarders $(\mathcal{S F})$ : each node maintains a table containing the wake-up schedules of its forwarders, so the transmissions are done when the intended forwarders are active. As detailed later in this section, the forwarders are selected so that their level $\mathcal{N}_{\mathcal{L}}$ is lower than the level of the node selecting them.

Hereinafter, we introduce the steps of our scheme.

\section{A. Initialization phase}

As soon as the sensor nodes are deployed on the network, the sink initiate the construction phase by broadcasting a construct topology packets $(\mathrm{CT})$. Among its parameters, the CT packet includes $\mathcal{N}_{\mathcal{L}}=0$. Upon receiving the construct packet, sink's neighbors within its transmission range scan the packet parameters. Then, prepare their own $\mathrm{CT}$ packets by incrementing the $\mathcal{N}_{\mathcal{L}}$, and adding their $\mathcal{D C S}$ then broadcast to their neighbors. While receiving all the CT packets, each node scans the parameters and considers as forwarders the nodes having the least $\mathcal{N}_{\mathcal{L}}$. They can select one or more forwarders (with the same $\mathcal{N}_{\mathcal{L}}$ ).

The initialization phase is made in multiple rounds. Each round corresponds to a different transmission power level. Therefore, in a given round, a node considers the CT packets from new potential forwarders and it has also to update the necessary TPL of some forwarders if it receives their $\mathrm{CT}$ packets in other rounds.

The expected goals of transmission power control in this scheme are: sleep latency reducing and energy saving. At the end of this phase, the network is layered and each node holds a set of forwarders with their schedules and the suitable transmission power level to communicate with them.

\section{B. Steady-state phase}

In this phase, generated or received packets are forwarded hop by hop to the sink. To this end, any potential sender refers to its $\mathcal{S F}$ table to choose one forwarder. To avoid high sleep latencies, a sender prefers the upcoming to wake up forwarder.

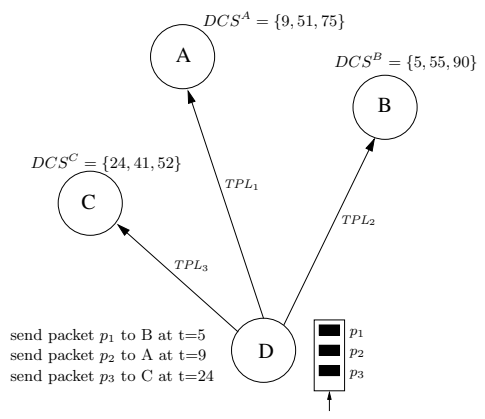

Figure 2. Periodic schedule with a duty-cycle of $3 \%$.

Let us consider the example where a node $D$ has selected three forwarders: $A, B$, and $C$ associated respectively to three TPLs. Thus, we put $\mathcal{S} \mathcal{F}_{\mathcal{D}}=\{\mathcal{A}, \mathcal{B}, \mathcal{C}\}$. As shown in Fig. 2, we assume that the packets $p_{1}, p_{2}$ and $p_{3}$ arrive respectively before the time-slots 5,9 , and 24 . Now, taking into account the active time-slots of its forwarders, D can prepare a packet forwarding sequence. Thus, $p_{1}, p_{2}$, and $p_{3}$ will be sent respectively to $B, A$, and $C$ in the time-slots 5,9 , and 24 .

\section{Performance Evaluation}

In order to establish whether the proposed scheme really has a positive impact on the end-to-end delay and with the purpose of setting the best value for certain parameters, we have turned to the CASTALIA Simulator [7].

\section{A. Simulation Setup}

In our simulations, we randomly deploy sensor nodes in a square area. Concerning the transmission power, we have chosen three transmission power levels: 31,23 , and 15 corresponding to respectively $(0 \mathrm{dBm},-3 \mathrm{dBm}$, and $-7 \mathrm{dBm})$. The performance metrics in our study are as follows: 
Delay: we measure the delay as the time elapsed from the packet sending by a source until it reaches the sink. Due to the random distribution of the sleep latencies we use the average delay as a network performance metric.

Energy consumption: we also measure the energy consumed by the packet transmitting from its sending by a source until it reaches the sink.

\section{B. Comparison}

We compare our design with a traditional scheme based on shortest-path routing (SPR). Unlike our scheme, in SPR all the nodes run with maximum transmission power and select only one forwarder (the closest one to the sink in terms of number of hops). To understand the behavior of our design under more realistic scenarios, we now examine the effect of different node densities on the average end-to-end delay. To this end, we randomly deploy 800 nodes on a square area with a side length varying from $200 \mathrm{~m}$ to $400 \mathrm{~m}$. The nodes operate with a duty-cycle of $3 \%$.

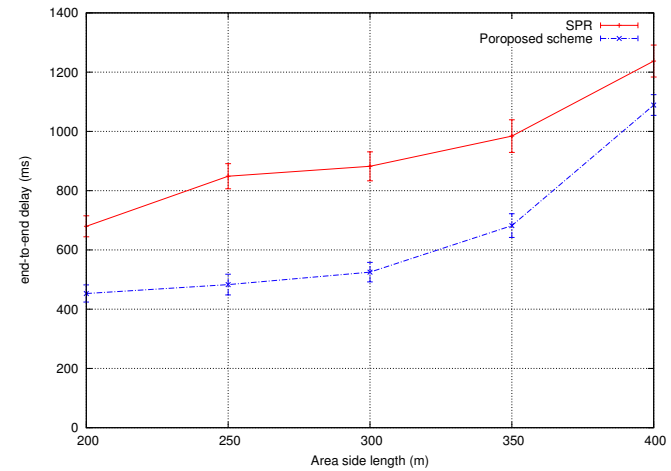

Figure 3. Delay vs. density (Network size $=800$ nodes).

To better understand how our approach behaves in these conditions, figure 3 plots the results of the average end-to-end delay evolution. As first result, we can see that the higher the density of nodes, the lower the average delay. Our approach is better because each node has more opportunities to send and consequently reduces the sleep latency. We note a significant difference in favor of our approach except two cases where the gain is less important. The first case concerns the side length $200 \mathrm{~m}$. Here the deployment is denser and although our approach is better, but the gain is less significant. The reason is the large number of the selected forwarders with the low power transmission levels. In this case, the number of hops to reach the sink increases. Unlike the second case where the deployment is sparse (side length $400 \mathrm{~m}$ ), the nodes have much less potential forwarders especially with low TPLs. Thus, both approaches operates almost with the maximum TPL. Despite that, our approach remains better since the nodes take into account all the potential forwarders for each power level in contrast to SPR where a single forwarder is chosen.

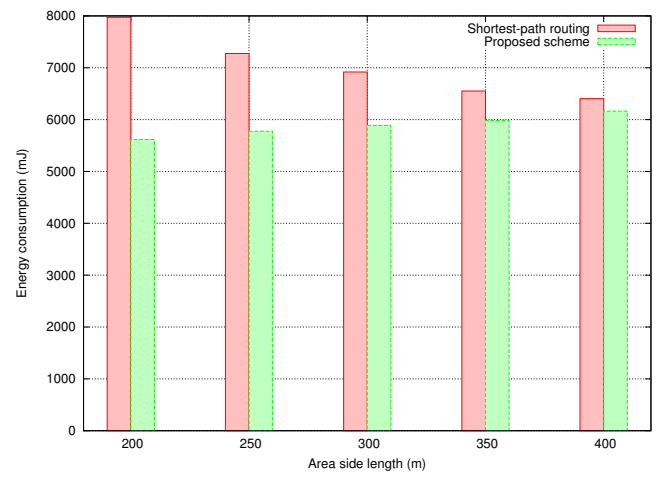

Figure 4. Energy cost vs. density (Network size $=800$ nodes).

It is interesting to observe in Fig. 4 the gain of energy in favor of our approach. Compared to SPR, our scheme saves between $7 \%$ and $30 \%$ according to the density. The explanation of this gain is the existence of many forwarders allowing often the use of low and suitable transmission power levels. Moreover, with high densities, the gain is more important because with SPR the interference radius is larger, so we noticed many retransmissions. Furthermore, although the consumed energy varies differently for the two algorithms, the results converge on the low densities because our approach behaves as SPR.

\section{CONCLUSION}

In this paper, we study the potential advantages and limitations of dynamic and delay-aware forwarder selection in multihop WSN. Moreover, we base our design on the transmission power control as a mean to select all the potential forwarders on one hand, and to save energy by reducing the packet transmission cost on other hand. Through extensive simulations, we examined the performance of our design under different conditions and demonstrated that it outperforms the traditional shortest path routing in terms of end-to-end communication delay and energy cost.

\section{REFERENCES}

[1] Y. Gu and T. He, "Dynamic Switching-Based Data Forwarding for Low-Duty-Cycle Wireless Sensor Networks", In IEEE Transactions on Mobile Computing, vol.10, no.12, pp.1741-1754, Dec. 2011.

[2] Y. Gu, T. He, M. Lin and J. Xu, "Spatio-temporal Delay Control for Low-Duty-Cycle Sensor Networks", In Proceedings of IEEE Real-Time Systems Symposium (RTSS'09), 2009.

[3] S. Lin, J. Zhang, G. Zhou, L. Gu, T. He and J. A. Stankovic, "ATPC: Adaptive Transmission Power Control for Wireless Sensor Networks", In Proceedings of ACM Conference on Embedded Networked Sensor Systems (SenSys'06), 2006.

[4] J. Polastre and D. Culler. Versatile low power media access for wireless sensor networks. In Proceedings of ACM Conference on Embedded Networked Sensor Systems (SenSys'04), 2004.

[5] F. Wang and J. Liu, "On Reliable Broadcast in Low Duty-Cycle Wireless Sensor Networks", In IEEE Transactions on Mobile Computing, vol. 11, no. 5, pp. 767-779, 2012.

[6] W. Ye, F. Silva, and J. Heidemann, "Ultra-low dudty cycle mac with scheduled channel polling", in Proceedings of ACM Conference on Embedded Networked Sensor Systems (SenSys'06), 2006.

[7] Castalia Simulator website, http://castalia.npc.nicta.com.au/. 